\title{
Headache and MTHFRT677T Genotype in a Young Woman - Clinical Case
}

\author{
Damelan Kombate ${ }^{1 *}$, Sirui Zhou ${ }^{2}$, Seylom Kossi Biosse ${ }^{3}$, Agbémélé K \\ M Soedje $^{3}$, Kossivi Apetse ${ }^{3}$, Komi Assogba ${ }^{3}$, Koffi AA Balogou ${ }^{3}$ and Guy \\ A Rouleau ${ }^{2}$ \\ ${ }^{1}$ Department of Neurology, University of Kara, Togo \\ ${ }^{2}$ Teaching Hospital Campus, Neurology Department, University of Lomé, Togo \\ ${ }^{3}$ Montreal Neurological Institute and Hospital, Department of Neurology and \\ Neurosurgery, McGill University, Montreal, QC, Canada \\ *Corresponding Author: Damelan Kombate, Department of Neurology, University \\ of Kara, Togo.
}

Received: June 20, 2021

Published: November 12, 2021

(C) All rights are reserved by Damelan

Kombate., et al.

\begin{abstract}
Introduction: The MTHFRC677T polymorphism is involved in several pathologies.

Objectives: We report one case of headache associated with the MTHFR T677T genotype in a woman.

Observation: A 39-year-old woman was admitted in neurology department on March 12, 2012 for headaches with feet and hands parathesia for several months. The physical examination had noted a decrease in osteotendinous reflexes and an abolition of the plantar cutaneous reflex. Hamilton's 17-item depression score was 7. In the etiological investigation of probable polyneuropathy, homocysteine was $88.39 \mu \mathrm{mol} / \mathrm{l}$ (standard < $15 \mu \mathrm{mol} / \mathrm{l}$ ), folates $2.34 \mathrm{nmol} / \mathrm{l}$ (standard $\geq 11 \mathrm{nmol} / \mathrm{l}$ ) and vitamin $\mathrm{B}_{12} 132 \mathrm{pmol} / \mathrm{l}$ (standard $\geq 147 \mathrm{pmol} / \mathrm{l}$ ). The aetiological investigation of this hyperhomocysteinemia revealed an MTHFRT677T genotype.

Conclusion: The MTHFRT677T genotype is associated with a variety of manifestations ranging from metabolic ( $\mathrm{B}_{12}$ deficiency), headache and polyneuropathies.
\end{abstract}

Keywords: MTHFR Polymorphism; Headache; Polyneuropathy

\section{Introduction}

There are multiple polymorphisms of the methylene tetrahydrofolate reductase (MTHFR) gene. The MTHFRC677T polymorphism including the MTHFRT677T genotype induces a significant deficiency in the activity of the enzyme MTHFR which compromises the conversion of homocysteine into methionine [1]. Homocysteine metabolism involves vitamins $\mathrm{B}_{6}, \mathrm{~B}_{9}$, and $\mathrm{B}_{12}$. The $M T H$ FRT677T genotype has been associated with vitamin $\mathrm{B}_{12}$ deficiency. Vitamin $B_{9}$ deficiency may also be linked to several causes, including nutritional factors [2]. The MTHFRC677T genotype is described in vascular diseases through Hyperhomocysteinemia, in other neurological conditions such as migraine, peripheral neuropathies and psychiatric conditions such as depression [2].

\section{Objectives}

We report one case of headache associated with the homozygous MTHFR T677T genotype in a woman.

\section{Case Report}

A 39-year-old patient was admitted in neurology department on March 12, 2012 for headache, paraesthesia on the feet and hands 
for several months. The headaches were sometimes occipital or in whole head, with a sensation of heaviness, burning head almost permanent, without nausea or vomiting, not calmed by antalgic drugs. Headaches were rated at $40 / 100$ on the analog visual scale. Headaches were associated with permanent vertigo, an impression of lightness with unstable walking. She sometimes had sleep paralysis. The patient had a depressive episode in 2009 that had regressed after 6 months with psychiatric care. Before her admission in neurology department, the patient had undergone several treatment including vitamin B complexes, which resulted in significant regression of paresthesia of feet and hands. In her background, she has 6 children, including a 24-year-old asthmatic girl, and a 14-year-old epileptic boy. She has a 28-year-old (maternal) half-sister with chronic headaches, a 38-year-old half-brother with chronic psychosis, a 34-year-old half-brother with eye pain, and a 43-year-old half-brother with sensory polyneuropathies. At the physical examination, the blood pressure was 120/80 $\mathrm{mm} \mathrm{Hg}$ in both arms, the weight was $75 \mathrm{~kg}$ for a height of $1 \mathrm{~m} 69$ (Body Mass Index $=26.2 \mathrm{~kg} / \mathrm{m}^{2}$ ). There was neither deep nor superficial sensory disturbance. The osteotendinous reflexes were diminished and the cutaneo-plantar reflexes were abolished. There was no nystagmus. The rest of the physical examination was normal. The patient was assessed by 2 psychiatricians who concluded that she was not depressed. Two evaluations with an interval of two weeks of Hamilton scale at 17 items [3] displayed an average score of 7. The biologic test including creatinine, blood glucose, total cholesterol, HDL, LDL, triglycerides, transaminases and Gamma Glutamyl Transferases (Gamma GT) were normal. Blood count, C-reactive protein (CRP), erythrocyte sedimentation rate, thyroid hormones and blood ionogram were normal. Human immunodeficiency virus (HIV) serology was negative. In the aetiological investigation of probable polyneuropathy, homocysteine was $88.39 \mu \mathrm{mol} / \mathrm{l}$ (standard $<15 \mu \mathrm{mol} / \mathrm{l}$ ), folates $2.34 \mathrm{nmol} / \mathrm{l}$ (standard $\geq 11 \mathrm{nmol} / \mathrm{l}$ ) and vitamin $\mathrm{B}_{12}, 132 \mathrm{pmol} / \mathrm{l}$ (standard $\geq 147 \mathrm{pmol} / \mathrm{l}$ ). The high level of homocysteinemia associated with vitamin folates and $\mathrm{B}_{12}$ deficiencies motivated the investigation of the polymorphism $\mathrm{MTH}$ FRC677T. For the genotyping, the patient had given verbal consent. The MTHFRC677T polymorphisms were genotyped by TaqMan SNP genotyping tests according to the manufacturer's instructions. The genotype was called using QuantStudioTM 7 Flex (v 1.0) (Applied Biosystem) real-time PCR system and software on DNA samples extracted from saliva samples. The search for this polymorphism displayed a homozygous genotype MTHFRT677T. The electroneuromyogram (Figure 1), Electroencephalogram (figure 3), and brain CT scanner were normal. We have retained the diagnosis of MTHFRT677T genotype associated with chronic headaches by somatization disorders according to the criteria of the International Headache Society [3] with probable polyneuropathy according to the medical history and biological findings. She was treated with $10 \mathrm{mg}$ per day of folic acid, $250 \mu \mathrm{mol} / \mathrm{l}$ of vitamin $\mathrm{B}_{12}$ for 6 months and 10 drops of amitriptyline in the evening for three months. After 6 months of treatment, the headaches had regressed to 15/100 with much more spaced intervals. The biologic control test of folate was $45.4 \mathrm{nmol} / \mathrm{l}$, the vitamin $\mathrm{B}_{12}$ was $449 \mathrm{pmol} / \mathrm{l}$ and homocysteine was $33.03 \mu \mathrm{mol} / \mathrm{l}$. She was diagnosed open-angle glaucoma in 2016 and treated with a beta-blocker (timolol) with normalization of intraocular pressure. Since July 2020, she has had tinnitus of motor-sound type in both ears with a slight hearing impairment of the right ear on the audiogram (Figure 2).
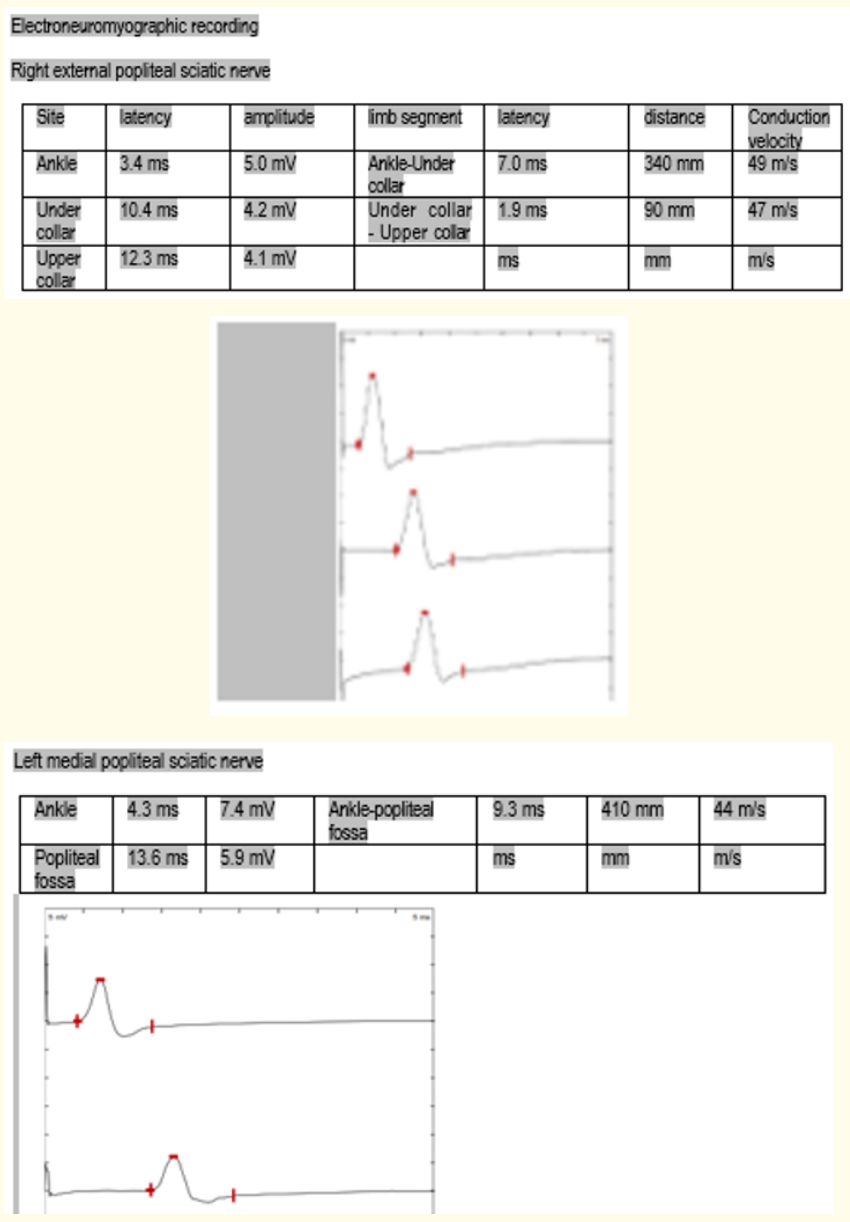


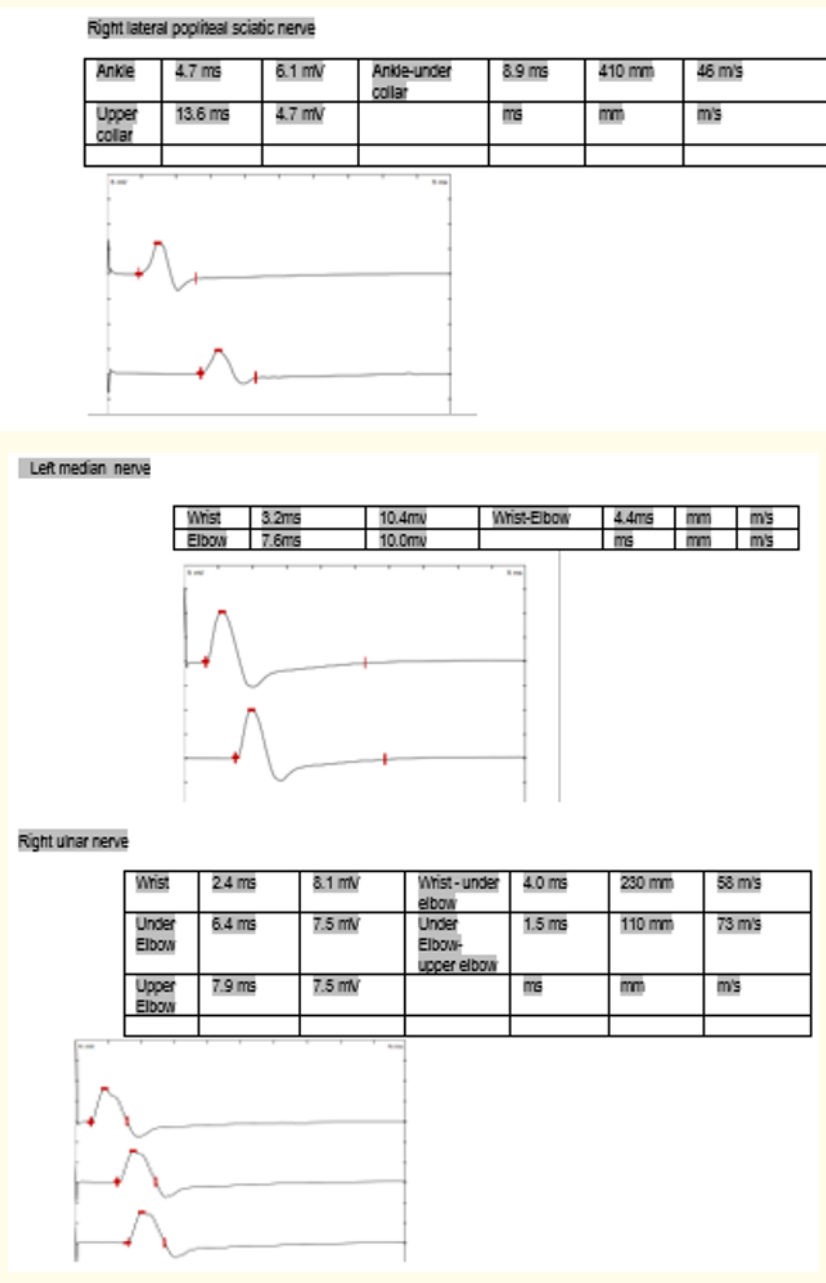

\section{F wave}
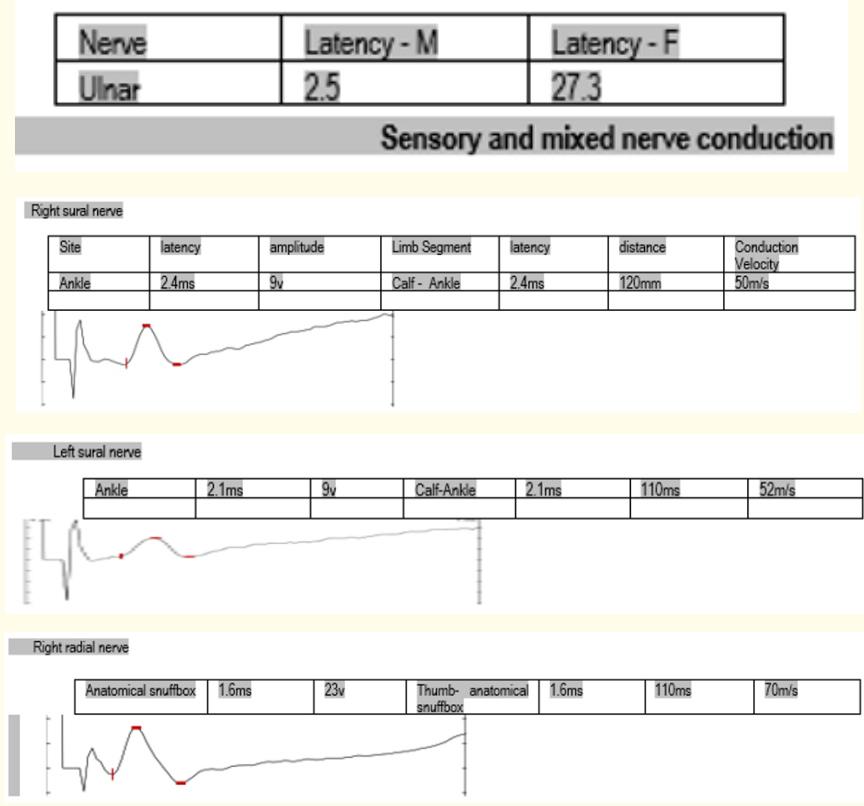

Figure 1: Normal electroneuromyography.

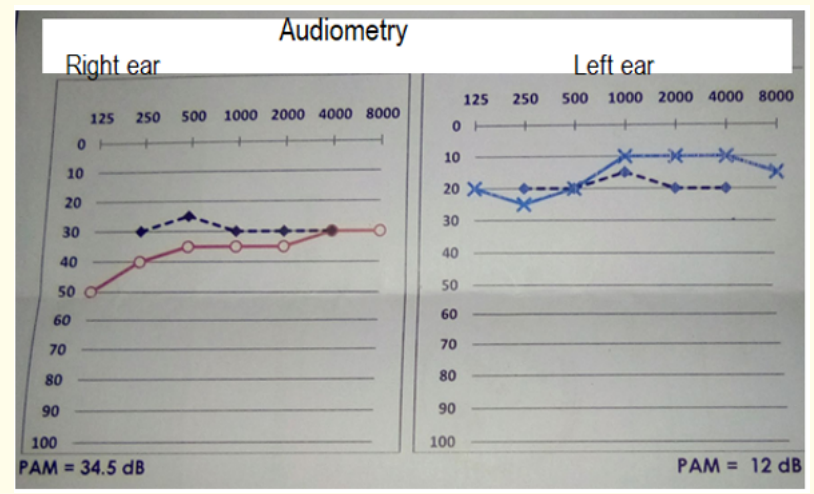

Figure 2: Audiometry showing a mild right-sided hearing loss on the low frequencies.

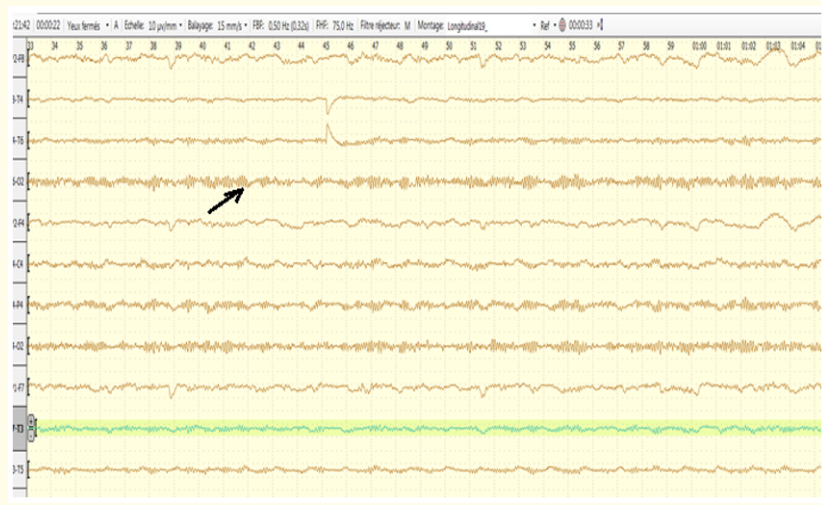

Figure 3: Normal EEG pattern with alpha posterior dominant rhythm.

\section{Discussion}

The MTHFRT677T genotype was described in vascular pathologies such as hypertension, in psychiatric conditions such as depression and migraine. This clinical case raises the multiplicity of pathologies involving the MTHFR gene. Although the majority of the literature associates the MTHFRT677T genotype with migraine, the headaches in this clinical case raises the characteristics of psychogenic headaches [4]. Recent studies have pointed out the association of a migraine without aura and the MTHFRC677T polymorphism in its homozygous or heterozygous form [5]. However, the headaches in our patient did not have the diagnostic criteria for migraines. Homocysteinemia remained high (33.03 $\mu \mathrm{mol} / \mathrm{l})$ despite the normalization of folate $(45.4 \mathrm{nmol} / \mathrm{l})$ and vitamin $\mathrm{B}_{12}$ (449 pmol/l), which ruled out vitamin deficiency as the cause of hyperhomocysteinemia. In homozygous TT forms of the MTHFR gene, the enzymatic activity of MTHFR is no longer sufficient to ensure normal homocysteinemia [6]. Glaucoma is one of the most common ophthalmic conditions with progressive atrophy of the 
optic nerve and loss of vision. Multiple meta -analyses have established the correlation between polymorphism of MTHFRC677T and open-angle glaucoma, however the pathophysiological mechanism remains poorly elucidated and a vascular cause including hyperhomocysteinemia is suspected [7]. We do not have sufficient arguments for the diagnosis of sensitive polyneuropathy in this patient. However, folate-deficiency neuropathy is reportedly characterized by a predominance of sensory symptoms with a slow progression. This study shows the importance of folate deficiency in the differential diagnosis of neuropathy, in our developing countries. Hyperhomocysteinemia is described in diabetes-related neuropathy with a complex pathophysiology [8]. Hyperhomocysteinemia is known as a predisposing factor for peripheral neuropathy may be directly or indirectly through the folate and vitamin $\mathrm{B}_{12}$ deficiencies, two factors that provide peripheral neuropathy. The $\mathrm{B}_{12}$ deficiency described in Biermer's disease gives a picture of combined sclerosis of the spinal cord with pyramidal symptoms and ataxic sensory [9] Several studies have established the link between the MTHFRT677T genotype and vitamin $\mathrm{B}_{12}$ deficiency, but the mechanism of action is poorly understood. Cobalamin participates in biochemical reactions to the constitution of the basic protein of myelin and in the production of tetrahydrofolates for the synthesis of DNA [10]. If the folate deficiency in our patient is probably nutritional, the less common $\mathrm{B}_{12}$ deficiency in the population would be related to the homozygous genotype MTHFRT677T [11-13]. Folic acid may interfere with the synthesis of myelin. There is an interdependence between vitamin $\mathrm{B}_{12}$ and folic acid.

\section{Conclusion}

The MTHFRT677T genotype is associated with a variety of manifestations ranging from metabolic ( $B_{12}$ deficiency), headaches, polyneuropathies and psychiatric conditions. MTHFR T677T genotype greatly reduces the enzymatic activity of MTHFR. Hyperhomocysteinemia due to MTHFRT677T genotype cannot be reduced by vitamin B supplementation.

\section{Bibliography}

1. Haruki Koike., et al. "Clinicopathologic features of folate-deficiency neuropathy”. Neurology 84.10 (2015): 1026-1033.

2. Sechi GP., et al. "Clinicopathologic features of folate-deficiency neuropathy”. Neurology 85.12 (2015): 1090-1091.

3. Bech P. "Fifty years with the Hamilton scales for anxiety and depression. A tribute to Max Hamilton". Psychotherapy and Psychosomatics 78.4 (2009): 202-211.
4. Kobayashi Y., et al. "Severe leukoencephalopathy with cortical involvement and peripheral neuropathy due to FOLR1 deficiency". Brain Development 39.3 (2017): 266-270.

5. Bruce SG and Young TK. "Prevalence and risk factors for neuropathy in a Canadian First Nation community". Diabetes Care 31 (2008): 1837-1841.

6. Ambrosch A., et al. "Relation between homocysteinaemia and diabetic neuropathy in patients with Type 2 diabetes mellitus". Diabetic Medicine 18 (2001): 185-192.

7. Cohen JA., et al. "Increasing homocysteine levels and diabetic autonomic neuropathy". Autonomic Neuroscience 87 (2001): 268-273.

8. González R., et al. "Plasma homocysteine levels are independently associated with the severity of peripheral polyneuropathy in type 2 diabetic subjects". Journal of the Peripheral Nervous System 17 (2012): 191-196.

9. Jianbo L., et al. "Association of homocysteine with peripheral neuropathy in Chinese patients with type 2 diabetes". Diabetes Research and Clinical Practice 93 (2011): 38-42.

10. Luo JJ., et al. "Elevated plasma level of homocysteine is an independent risk factor for peripheral neuropathy". British Journal of Medicine and Medical Research 4 (2014): 161-169.

11. Herrmann W., et al. "Review of the role of hyperhomocysteinemia and B-vitamin deficiency in neurological and psychiatric disorders--current evidence and preliminary recommendations". Fortschritte der Neurologie · Psychiatrie 75 (2007): 515527.

12. Ansari R., et al. "Hyperhomocysteinemia and Neurologic Disorders: a Review". Journal of Clinical Neurology 10 (2014): 281-288.

13. Tozzi E., et al. "The Headaches and Polymorphisms of the Methylenetetrahydrofolate Reductase". Journal of Headache and Pain Management 2 (2017): 12.

Volume 4 Issue 12 December 2021

(C) All rights are reserved by Damelan Kombate., et al. 